\title{
Correction to: Parameter Development via In Situ Residual Stress Measurement and Post-deposition Analysis of Cold Spray CuNi Coatings
}

\author{
Robert F. Brown' ${ }^{1}$ Gregory M. Smith ${ }^{2} \cdot J_{0 h n}$ Potter $^{1} \cdot$ Timothy J. Eden $^{1}$
}

Published online: 30 July 2021

(C) ASM International 2021

Correction to: J Therm Spray Tech (2020) 29:1876-1891

https://doi.org/10.1007/s11666-020-01109-3

In the Discussion section, the Table 4 that appeared is a duplicate of Table 5.

Table 4 should read as follows:

Table 4 Coating property data comparison to surface preparation/deposition angle and coating adhesion

\begin{tabular}{lllccccc}
\hline Coating & \multicolumn{1}{c}{$\begin{array}{c}\text { Surface preparation \& } \\
\text { nozzle angle }\end{array}$} & $\begin{array}{c}\text { Deposition } \\
\text { thickness, mm }\end{array}$ & $\begin{array}{c}\text { Surface profile, } \\
\text { Ra } \mu \mathrm{m}\end{array}$ & $\begin{array}{c}\text { Hardness, } \\
\text { HV0.3 }\end{array}$ & $\begin{array}{c}\text { Porosity, } \\
\%\end{array}$ & $\begin{array}{c}\text { Adhesion, } \\
\text { MPa }\end{array}$ & $\begin{array}{c}\text { Residual stress, } \\
\text { MPa }\end{array}$ \\
\hline C & Grit-blast \& $90^{\circ}$ & 0.60 (5 passes) & 5.8 & $197 \pm 11$ & $2.4 \pm 0.2$ & $5.8 \pm 1.2$ & $+15 \pm 5$ \\
D & Abrasive pad \& $40^{\circ}+90^{\circ}$ & $0.46(5$ passes) & 0.79 & $180 \pm 27$ & $2.9 \pm 0.2$ & $44.4 \pm 4.5$ & $-19 \pm 5$ \\
E & Grit-blast \& $40^{\circ}+90^{\circ}$ & 0.25 (5 passes) & 4.4 & N/A & $2.0 \pm 0.2$ & $20.7 \pm 4.0$ & $+2 \pm 5$ \\
\hline
\end{tabular}

The original article can be found online at https:// doi.org/10.1007/s11666-020-01109-3.

Robert F. Brown

rfb74@psu.edu

1 The Pennsylvania State University, 3075 Research Drive, State College, PA 16801, USA

2 Naval Research Laboratory, 4555 Overlook Ave SW, Washington, DC 20375, USA

Publisher's Note Springer Nature remains neutral with regard to jurisdictional claims in published maps and institutional affiliations. 